\title{
Expressão de CDX2 e mucinas (MUC1, MUC2, MUC5AC e MUC6) em esôfago de Barrett antes e após fundoplicatura de Nissen
}

Primeira submissão em 03/02/12 Última submissão em 01/07/12 Aceito para publicação em 08/07/12 Publicado em 20/10/12

\section{Expression of CDX2 and mucins (MUC1, MUC2, MUC5AC and MUC6) in Barrett's esophagus before and after Nissen fundoplication}

Luciana Rodrigues de Meirelles'; Thiago Pires Brito²; Ana Claudia Sparapani Piaza ${ }^{3}$; Luiz Roberto Lopes ${ }^{4}$

\begin{abstract}
unitermos
Esôfago de Barrett

Mucinas

CDX2

Imuno-histoquímica

Doença do refluxo gastroesofágico

Fundoplicatura de Nissen

\section{resumo}

Introdução: O esôfago de Barrett (EB) corresponde à substituição do epitélio escamoso por um do tipo intestinal, em resposta ao refluxo crônico nos pacientes com doença do refluxo gastroesofágico (DRGE). É um importante precursor do adenocarcinoma esofágico. A fundoplicatura de Nissen (FN) é uma cirurgia antirrefluxo que visa a reduzir a agressão à mucosa esofágica. Alterações no padrão de expressão imuno-histoquímica de mucinas e de CDX2 no EB antes e depois da FN podem ser úteis na identificação de um padrão de expressão desses marcadores e, eventualmente, na identificação de casos com risco de evolução para malignidade. Objetivos: Avaliar e comparar a imunoexpressão de CDX2 e mucinas no EB de pacientes com DRGE submetidos à FN antes e após a cirurgia. Materiais e métodos: Estudo retrospectivo de 25 pacientes com diagnóstico de DRGE e EB submetidos à $\mathrm{FN}$, acompanhados por, pelo menos, três anos. Foram feitos análise histológica e estudo imuno-histoquímico das biópsias endoscópicas antes e após a cirurgia, comparando-se a inflamação e a imunoexpressão de MUC1, MUC2, MUC5AC, MUC6 e CDX2. Estimou-se a porcentagem de células com expressão para os marcadores estudados na mucosa de Barrett: 0\%-25\%, 25\%-75\% e 75\%-100\% das células positivas. Foram utilizados os testes de McNemar e Stuart-William e adotou-se o nível de 5\% de significância estatística. Resultados e conclusão: Não houve diferenças significativas quanto a presença ou intensidade de inflamação, nem da imunoexpressão de mucinas e CDX2 no EB antes e após a FN. O tratamento cirúrgico não influenciou a mudança da expressão dessas glicoproteínas no EB.
\end{abstract}

\section{abstract}

Introduction: Barrett's esophagus (BE) is characterized by the exchange of esophageal squamous epithelium for intestinal type in response to chronic reflux in patients with gastroesophageal reflux disease (GERD).It is an important precursor of esophageal adenocarcinoma. Nissen fundoplication (NF) is an antireflux surgery which aims to reduce esophageal mucosa inflammation. Changes in the immunohistochemical expression patterns of mucins (MUC1, MUC2, MUC5AC and MUC6) and CDX2 in BE before and after NF may be useful to identify the expression patterns of these markers and, possibly, to detect cases with risks of malignancy. Objectives: To investigate and compare mucin and CDX2 immunoexpression in BE patients with GERD before and after NF. Material and methods: This retrospective study comprised 25 patients with GERD and BE who had been submitted to NF. The patients had a 3-year minimum follow up. Histological and immunohistochemical analyses of endoscopic biopsies were performed before and after the surgery, comparing inflammation and MUC1, MUC2, MUC5AC, MUC6 and CDX2 immunoexpression. The percentage of Barrett mucosa cells with expression to the studied markers was estimated at 0\%-25\%, 25\%-75\% and 75\%-100\%. McNemar and Stuart-William tests were used and the significance level of $<0.05$ was applied. Results and conclusion: Concerning the presence or the intensity of inflammation and mucin and CDX2 expression in $B E$, there were no significant differences before and after NF. The surgical procedure did not promote any changes in the expression of these glycoproteins in $B E$. key words

Barrett's esophagus

Mucins

CDX2

Immunohistochemistry

Gastroesophageal reflux disease

Nissen fundoplication

\footnotetext{
1. Doutora; professora do Departamento de Anatomia Patológica, área de Patologia do Trato Castrointestinal, da Faculdade de Ciências Médicas da Universidade Estadual de Campinas (UNICAMP).

2. Residente no Departamento de Otorrinolaringologia da Faculdade de Ciências Médicas da Universidade Estadual de Campinas (UNICAMP).

3. Bióloga do Laboratório de Pesquisa da Pós-graduação do Departamento de Anatomia Patológica da Faculdade de Ciências Médicas da UNICAMP.

4. Doutor; professor livre-docente associado do Departamento de Cirurgia da Faculdade de Ciências Médicas da UNICAMP.
} 


\section{Introdução}

O esôfago de Barrett (EB) é uma das principais complicações da doença do refluxo gastroesofágico de longa duração. Consiste em um processo peculiar de substituição do epitélio escamoso do esôfago por um epitélio colunar metaplásico do tipo intestinal(26). A metaplasia é considerada lesão pré-neoplásica, com imprevisível evolução para adenocarcinoma. Alguns estudos estimam que haja risco de até 50 vezes de desenvolvimento neoplásico sobre uma metaplasia decorrente de refluxo, quando comparado com a população geral ${ }^{(26,29)}$.

A doença do refluxo gastroesofágico (DRGE) é definida como uma afecção crônica decorrente do fluxo retrógrado do conteúdo gastroduodenal para o esôfago e/ou órgãos adjacentes a ele, acarretando um espectro variável de sintomas e/ou sinais esofagianos e/ou extraesofagianos, associados ou não a lesões teciduais ${ }^{(10,15,19)}$.

Há marcada correlação entre o tempo de duração dos sintomas da DRGE e o aumento do risco para o desenvolvimento de esôfago de Barrett e adenocarcinoma do esôfago $^{(21,22)}$. O tratamento pode ser clínico, o mais utilizado, e/ou cirúrgico. Neste último caso, a fundoplicatura de Nissen (FN) é o método de primeira escolha ${ }^{(8)}$.

A DRGE e suas complicações apresentam alta prevalência e também têm apresentado aumento de sua incidência, o que justifica sua grande importância na prática clínica. $O$ EB ocorre em cerca de $10 \%$ dos pacientes com DRGE e em $1,6 \%$ da população geral(27). A incidência de EB no Gastrocentro da Universidade Estadual de Campinas (UNICAMP) entre os pacientes com refluxo esofágico foi de 3,57\%, totalizando 22,4 casos $/ 100.000$ habitantes $^{(3)}$.

A importância biológica do EB é o risco de progressão para adenocarcinoma e a displasia é, até então, o marcador biológico preditivo dessa progressão. Embora muitas alterações genéticas e epigenéticas tenham sido descritas na sequência EB-displasia-adenocarcinoma, não há um marcador biomolecular preditivo utilizado na prática diagnóstica ${ }^{(20,25)}$.

Um grande avanço para o diagnóstico da origem tecidual de muitas neoplasias se deu com a descoberta das mucinas. Estas (MUC1, MUC2, MUC3, MUC4, MUC5AC, MUC5B e MUC6) compreendem um grupo de glicoproteínas que conferem propriedades viscoelásticas à camada de muco secretado pelas glândulas submucosas, oferecendo proteção a agressões externas (por exemplo, exposição ácida) e lubrificação ao órgão onde se situam ${ }^{(11)}$. Dividem-se as mucinas em dois grandes grupos: secretadas em forma de gel para o meio extracelular (MUC2, MUC5AC, MUC5B e MUC6) e mucinas de membrana (MUC1, MUC3 e MUC4) $)^{(4)}$.

O epitélio esofágico habitual e o epitélio de Barrett apresentam padrões descritos de expressão dessas glicoproteínas ${ }^{(23)}$. O epitélio esofágico normalmente expressa MUC5B nas glândulas submucosas e MUC1 e MUC4 em sua porção escamosa. No EB, as células caliciformes são capazes de produzir e secretar essas glicoproteínas, as quais sofrem modificações para desempenhar seu importante papel citoprotetor diante de um refluxo crônico ${ }^{(9,11)}$. O epitélio de Barrett mostra expressão de MUC5AC e MUC3 na porção superficial do epitélio colunar, MUC2 nas células caliciformes e MUC6 na porção glandular mais profunda ${ }^{(4)}$.

Chinyama et al.(9) demonstraram negatividade de MUC1 na metaplasia intestinal, ao passo que Flucke et al. (12) mostraram expressão da mesma glicoproteína em adenocarcinomas originados do Barrett, o que poderia ser usado para diferenciar lesões displásicas de adenocarcinomas já instalados, assim como para prever a evolução daquelas lesões pré-neoplásicas para adenocarcinoma, de acordo com o grau de expressão desses marcadores.

Burjonrappa et a.(16) demonstraram aumento da expressão de MUC1 em adenocarcinomas com origem em Barrett. As mucinas secretoras (MUC5AC e MUC6), por sua vez, tiveram redução de sua expressão na progressão da mucosa de Barrett para displasia e adenocarcinoma.

Chinyama et al. ${ }^{(9)}$ também demonstraram expressão de MUC2 no epitélio de Barrett, alteração também encontrada em células neoplásicas. Já as mucinas secretadas (MUC5AC e MUC6) apresentaram subexpressão na progressão do $E B$ para adenocarcinoma, o que poderia representar menor proteção contra o suco gástrico e, consequentemente, maior risco de malignização.

Em um estudo de coorte com 333 pacientes com EB, Amano et al.(2) observaram expressão de MUC2 no epitélio metaplásico em um terço dos casos. Neste grupo estudado, a maioria era formada de homens com hérnia de hiato e displasia.

Glickman et al.(13) observaram notável redução da expressão de MUC1, MUC2 e MUC3 em pacientes com displasia em EB. Também observaram redução da expressão de MUC5AC e MUC6 nos casos de displasia de alto grau. Não houve diferenças significantes entre as áreas de displasia de baixo e alto grau.

Estudos apontam que a expressão de CDX2 está associada ao epitélio de Barrett e que essa expressão pode antecipar as alterações morfológicas observadas nesse 
epitélio ${ }^{(1,17)}$. Moons et al. ${ }^{(18)}$ observaram que o CDX2 é expresso no epitélio metaplásico, mas não no escamoso de pacientes com refluxo gastroesofágico.

O CDX2 parece ter uma atuação como supressor tumoral na sequência EB-displasia-carcinoma com aumento de sua expressão na metaplasia intestinal, seguido de perda progressiva de expressão na evolução para adenocarcinoma ${ }^{(16,24)}$.

\section{Objetivos}

O objetivo do presente estudo foi avaliar a expressão imuno-histoquímica de CDX2 e mucinas (MUC1, MUC2, MUC5AC e MUC6) em EB de pacientes com diagnóstico de DRGE antes e após a realização de FN.

\section{Materiais e métodos}

Trata-se de um estudo retrospectivo de 25 pacientes acompanhados no Hospital das Clínicas da UNICAMP e no serviço de Endoscopia Digestiva do Gastrocentro no período de 1991 a 2005. Os critérios de inclusão para este estudo foram diagnóstico de DRGE; diagnóstico endoscópico e histológico de $\mathrm{EB}$; ter sido submetido ao tratamento cirúrgico da DRGE por meio da FN; acompanhamento clínico de, no mínimo, três anos a partir do diagnóstico de Barrett; e possuir biópsias pré e pós-cirúrgicas de controle.

As informações clínicas foram coletadas por meio da consulta dos prontuários médicos junto ao Serviço de Arquivo Médico (SAME) do Hospital das Clínicas da UNICAMP. O Comitê de Ética em Pesquisa da referida Faculdade aprovou a realização do projeto (parecer 761/2007), estando de acordo com a resolução CNS-MS 196/96.

As variáveis clinicopatológicas levantadas foram nome, idade, gênero, cor, tempo entre queixa clínica e diagnóstico de Barrett (endoscópico e histopatológico), tempo de seguimento pós-cirúrgico, recorrência da doença e doenças associadas (obesidade, etilismo, tabagismo, gastrite endoscópica, hérnia de hiato e infecção por $H$. pylori).

Classificou-se como Barrett "curto" quando as projeções do epitélio tinham menos que $3 \mathrm{~cm}$ de extensão acima da transição esôfago-gástrica, e como Barrett "longo" quando acima de $3 \mathrm{~cm}$.

Todas as biópsias foram revistas pelo mesmo patologista com confirmação do diagnóstico histológico de mucosa do tipo Barrett mediante a observação de epitélio colunar especializado com células caliciformes, caracterizado morfologicamente como metaplasia intestinal.

Avaliamos por meio de histologia convencional e também por meio de imuno-histoquímica duas biópsias em seu seguimento: a última biópsia antecedendo a realização da FN, representando as alterações histológicas crônicas provocadas pela DRGE no esôfago e a última biópsia em seu seguimento após a cirurgia, representando as alterações provocadas a mais longo prazo no epitélio de Barrett após a correção cirúrgica do refluxo. A presença de inflamação (crônica ou ativa) associada à metaplasia e a presença de neoplasia intraepitelial e/ou adenocarcinoma foram avaliadas à histologia convencional.

Os espécimes incluídos em parafina foram processados para imuno-histoquímica, utilizando-se os anticorpos primários CDX2 (BioGenex, código AM392-5M) 1:25, MUC1 (código NCL-MUC-1) 1:300, MUC2 (código NCL-MUC-2) 1:300, MUC5AC (código NCL-MUC-5AC) 1:300 e MUC6 (código NCL-MUC-6) 1:300, estes últimos procedentes do laboratório Novocastra. O bloqueio da peroxidase endógena foi feito em três banhos de $\mathrm{H}_{2} \mathrm{O}_{2}$ a 10 volumes, cada um com cinco minutos de duração, seguidos de lavagem em água corrente e água destilada. A recuperação antigênica foi feita pelo método de calor úmido, utilizando-se panela a vapor. Utilizou-se também tampão citrato de sódio $(\mathrm{pH}$ 6) e coquetel de polímeros (Novolink Max Polymer, Novocastra, RE7260-K). Para revelação, foram utilizadas solução de tetra-hidrocloreto de 3,5-diamino-benzidina (DAB) e contracoloração com hematoxilina de Harris.

Para controles positivos da reação imuno-histoquímica, utilizamos biópsias de carcinoma de mama com positividade em membrana da MUC1, biópsias de cólon com positividade citoplasmática de MUC2 em células caliciformes e nuclear de CDX2. Para controle positivo de MUC5AC e MUC6, utilizamos biópsias de estômago, com marcação citoplasmática das células mucosas da superfície e do colo das glândulas para o anticorpo da mucina MUC5AC, e marcação citoplasmática do epitélio gástrico profundo (células mucosas e principais) para o anticorpo da mucina MUC6.

Estimou-se a porcentagem de células com expressão positiva para os marcadores estudados (MUC1, MUC2, MUC5AC e MUC6) e CDX2 na mucosa de Barrett, tendo sido considerados todos os campos presentes em cada lâmina: 0\%-25\%, 25\%-75\% e 75\%-100\% das células analisadas positivas.

Os dados foram analisados descritivamente por frequências absolutas $(n)$ e relativas (\%). Foram utilizados os testes 
de McNeemar e Stuart-William para analisar as mudanças das variáveis clinicopatológicas após a FN.

Foi adotado o nível de $5 \%$ de significância estatística. A análise dos dados foi realizada por meio do software SAS, versão 9.1.3 (SAS Institute Inc., Cary, USA).

\section{Resultados}

O tempo médio entre o início da queixa clínica (pirose, disfagia e sintomas extraesofágicos, como tosse crônica, otite e faringite) e o diagnóstico endoscópico-histológico de EB foi de 88,96 meses (mediana de 49 meses), variando entre sete meses e 30 anos (Tabela 1).

As principais doenças gastrintestinais associadas ao EB foram gastrite confirmada pela histologia (64\%), hérnia de hiato (64\%) e infecção por H. pylori (44\%). Tabagismo (28\%) e etilismo (28\%) foram hábitos de vida também associados numa pequena porcentagem de casos (Tabela 1).

A recorrência da DRGE após o tratamento cirúrgico por FN, definida pela presença de sintomas clínicos de refluxo associada a sinais endoscópicos de esofagite de refluxo, foi verificada em cinco casos (20\%), com um tempo médio de retorno dos sintomas após 30,4 meses (variação: oito meses a cinco anos). Não houve diferenças estatisticamente significantes $(p=0,1967)$ quanto ao grau e à natureza da inflamação antes e após a FN (Tabela 2).

Antes da FN, observamos alterações displásicas e adenocarcinoma em apenas $24 \%$ dos pacientes estudados (cinco com displasia de baixo grau e um com displasia de alto grau e focos de adenocarcinoma). Não houve diagnóstico de novas lesões displásicas, nem tampouco progressão das lesões previamente diagnosticadas após a cirurgia (Tabela 3), ou seja, os achados no material examinado antes e após a FN são os mesmos.

As biópsias examinadas antes e após a FN continham epitélio da junção escamo-glandular esofágica com metaplasia intestinal, confirmando a mucosa de Barrett nas duas amostragens. A análise das mucinas e do CDX2 antes e após a FN foi feita na porção glandular desses espécimes.

Observamos positividade citoplasmática para MUC1 no epitélio escamoso esofágico na quase totalidade dos casos estudados e não houve diferenças significantes $(p=0,7389)$ de sua expressão antes e após a FN, como não houve positividade de MUC1 na mucosa glandular nem nas áreas de Barrett em nenhum dos casos estudados.
Características clinicoepidemiológicas

Tabela 1

dos pacientes estudados ${ }^{(25)}$

\begin{tabular}{|c|c|c|}
\hline Características & $n$ & $\%$ \\
\hline \multicolumn{3}{|c|}{ Sexo } \\
\hline Feminino & 8 & 32 \\
\hline Masculino & 17 & 68 \\
\hline \multicolumn{3}{|c|}{ Idade } \\
\hline Até os 63 anos & 13 & 52 \\
\hline Acima de 63 anos & 12 & 48 \\
\hline \multicolumn{3}{|c|}{ Tempo entre sintomas e diagnóstico } \\
\hline Até 49 meses & 13 & 52 \\
\hline Mais que 49 meses & 12 & 48 \\
\hline \multicolumn{3}{|c|}{ Cor } \\
\hline Branca & 20 & 80 \\
\hline Parda & 3 & 12 \\
\hline Preta & 2 & 8 \\
\hline \multicolumn{3}{|c|}{ Extensão endoscópica do Barret } \\
\hline Curta & 13 & 52 \\
\hline Longa & 12 & 48 \\
\hline \multicolumn{3}{|l|}{ Gastrite histológica } \\
\hline $\operatorname{Sim}$ & 16 & 64 \\
\hline Não & 9 & 36 \\
\hline \multicolumn{3}{|c|}{ H. Pylori } \\
\hline Sim & 11 & 44 \\
\hline Não & 14 & 56 \\
\hline \multicolumn{3}{|c|}{ Hérnia de hiato } \\
\hline Sim & 16 & 64 \\
\hline Não & 9 & 36 \\
\hline \multicolumn{3}{|c|}{ Tabagismo } \\
\hline Sim & 7 & 28 \\
\hline Não & 18 & 72 \\
\hline \multicolumn{3}{|c|}{ Consumo de álcool } \\
\hline Sim & 7 & 28 \\
\hline Não & 18 & 72 \\
\hline \multicolumn{3}{|c|}{ Obesidade } \\
\hline Sim & 2 & 8 \\
\hline Não & 23 & 92 \\
\hline
\end{tabular}

A positividade citoplasmática para MUC2 foi observada em células caliciformes do epitélio metaplásico de Barrett na quase totalidade dos casos (16/25) estudados. Sua expressão permaneceu homogeneamente distribuída após a realização da cirurgia ( $p=0,2117$; Tabela 4). 


\begin{tabular}{|c|c|c|c|c|}
\hline \multicolumn{5}{|c|}{ Inflamação pós } \\
\hline Inflamação pré & Ausente & Ativa & Crônica & Total \\
\hline Ausente & 2 & 3 & 1 & 6 \\
\hline Ativa & 4 & 2 & 5 & 11 \\
\hline Crônica & 5 & 0 & 3 & 8 \\
\hline Total & 11 & 5 & 9 & 25 \\
\hline
\end{tabular}

Valor de $p=0,1967$ (teste de Stuart-Maxwell)

FN: fundoplicatura de Nissen.

\begin{tabular}{|c|c|c|}
\hline Tabela 3 & \multicolumn{2}{|c|}{$\begin{array}{l}\text { Displasia/adenocarcinoma antes } \\
\text { ( } 25 \text { biópsias) e depois da FN ( } 25 \\
\text { biópsias) }\end{array}$} \\
\hline Características & \begin{tabular}{l|l} 
& $n$ \\
\end{tabular} & $\%$ \\
\hline \multicolumn{3}{|c|}{ Displasia/adenocarcinoma pré } \\
\hline Não & \begin{tabular}{|l|l|}
19 &
\end{tabular} & 76 \\
\hline Sim & 6 & 24 \\
\hline \multicolumn{3}{|c|}{ Displasia/adenocarcinoma pós } \\
\hline Não & \begin{tabular}{l|l}
19 & 19
\end{tabular} & 76 \\
\hline Sim & 6 & 24 \\
\hline
\end{tabular}

Valor de $p=$ não calculável

FN: fundoplicatura de Nissen.

\begin{tabular}{l|l|l|l|l|} 
Tabela 4 & $\begin{array}{l}\text { Porcentagem de células positivas para } \\
\text { MUC2 na mucosa de Barrett antes e } \\
\text { após FN }\end{array}$ \\
\hline MUC2 pré & \multicolumn{4}{|c|}{ MUC2 pós } \\
\hline & 0 & $0 \%-25 \%$ & $25 \%-100 \%$ & Total \\
\hline 0 & 9 & 0 & 4 & 13 \\
\hline $0 \%-25 \%$ & 4 & 3 & 1 & 8 \\
\hline $25 \%-100 \%$ & 1 & 1 & 2 & 4 \\
\hline Total & 14 & 4 & 7 & 25 \\
\hline
\end{tabular}

Valor de $p=0,2117$ (teste de Stuart-Maxwell)

FN: fundoplicatura de Nissen.

Quanto à imunoexpressão das mucinas secretoras (MUC5AC e MUC6), ambas com padrão citoplasmático de positividade, observamos distribuição com padrão similar ao descrito para a mucosa gástrica. A MUC5AC mostrou positividade citoplasmática no epitélio metaplásico e também na superfície luminal da mucosa colunar, enquanto a MUC6 mostrou positividade citoplasmática no epitélio metaplásico e na porção mais profunda das glândulas (Figuras 1 e 2). Não houve diferenças significantes na expressão de MUC5AC após a correção cirúrgica do refluxo (Tabela 5). Em relação à expressão do MUC6, observamos manutenção de sua expressão após a FN, porém, devido à casuística pequena, o valor de $p$ não foi calculável.

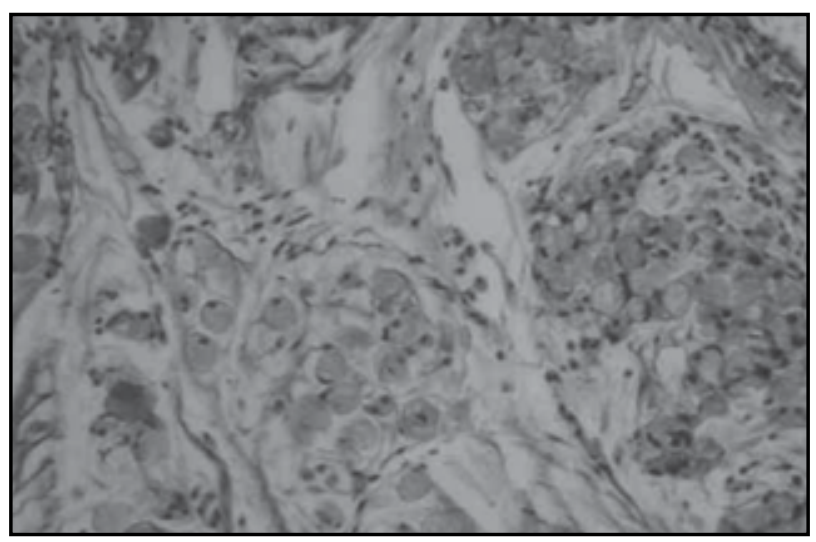

Figura 1 - Expressão citoplasmática de MUC5AC no epitélio metaplásico de Barrett e no epitélio colunar superficial em biópsia após FN (250x)

FN: fundoplicatura de Nissen.

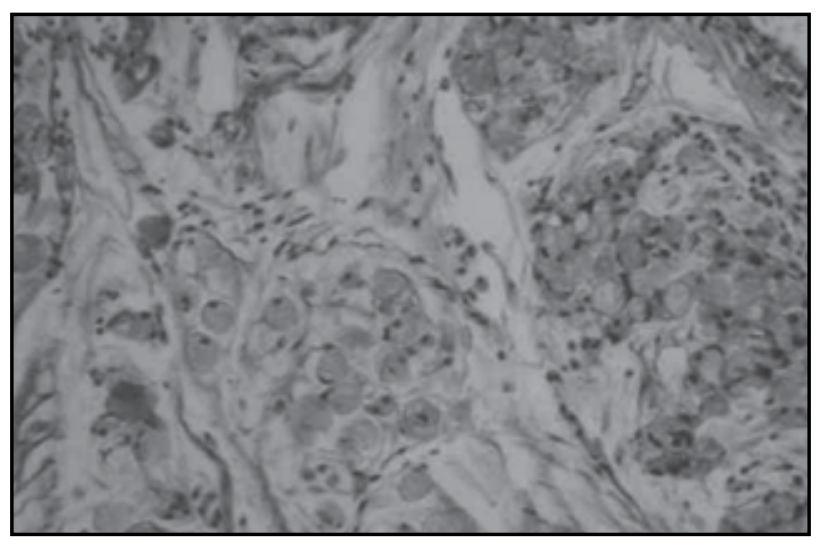

Figura 2 - Expressão citoplasmática de MUC6 no epitélio metaplásico de Barrett e na porção profunda do epitélio colunar em biópsia após FN (250x)

FN: fundoplicatura de Nissen.

\begin{tabular}{|l|c|c|c|c|} 
Tabela 5 5 & $\begin{array}{l}\text { Porcentagem de células positivas para } \\
\text { MUC5AC na mucosa de Barrett antes e } \\
\text { após FN }\end{array}$ \\
\hline $\begin{array}{c}\text { MUC5AC } \\
\text { pré }\end{array}$ & 0 & $25 \%-75 \%$ & $75 \%-100 \%$ & Total \\
\hline 0 & 5 & 1 & 6 & 12 \\
\hline $25 \%-75 \%$ & 0 & 0 & 3 & 3 \\
\hline $75 \%-100 \%$ & 2 & 2 & 6 & 10 \\
\hline Total & 7 & 3 & 15 & 25 \\
\hline
\end{tabular}

Valor de $p=0,2529$ (teste de Stuart-Maxwell)

FN: fundoplicatura de Nissen.

À semelhança da MUC2, o CDX2 também foi positivo nos núcleos do epitélio metaplásico de Barrett na quase totalidade dos casos estudados (16/25). Não observamos alterações de sua imunoexpressão após a FN $(p=0,4805$;

Tabela 6). 


\begin{tabular}{|c|c|c|c|c|}
\hline \multirow{3}{*}{$\begin{array}{l}\text { Tabela } 6 \\
\text { CDX2 pré }\end{array}$} & \multicolumn{4}{|c|}{$\begin{array}{l}\text { Porcentagem de células positivas para } \\
\text { CDX2 na mucosa de Barrett antes e } \\
\text { após FN }\end{array}$} \\
\hline & \multicolumn{3}{|c|}{ CDX2 pós } & \multirow{2}{*}{ Total } \\
\hline & 0 & $25 \%-75 \%$ & $75 \%-100 \%$ & \\
\hline 0 & 9 & 4 & 3 & 16 \\
\hline $25 \%-75 \%$ & 2 & 0 & 2 & 4 \\
\hline $75 \%-100 \%$ & 2 & 0 & 3 & 5 \\
\hline Total & 13 & 4 & 8 & 25 \\
\hline
\end{tabular}

\section{Discussão}

Houve um predomínio da população masculina, com proporção entre homens e mulheres de cerca de 2:1 (68\% e $32 \%$ aproximadamente). Os estudos epidemiológicos mostram que os sintomas de refluxo gastroesofágico ocorrem com frequência similar em homens e mulheres, ainda que o EB seja menos comum em mulheres por razões desconhecidas ${ }^{(5,27)}$. A média de idade dos pacientes com EB foi de 62 anos. Esses resultados corroboram a epidemiologia dos demais estudos da literatura ${ }^{(7)}$.

Os estudos em relação ao consumo de álcool e tabagismo são controversos. Van Sandick et al.(29) demonstraram maior prevalência de EB entre aqueles com história de tabagismo, ao passo que Chalasani et al. ${ }^{(8)}$ não evidenciaram associação entre metaplasia e uso de cigarro ou álcool. Nosso estudo mostrou associação com tabagismo e etilismo em $28 \%$ dos casos.

O tratamento cirúrgico do refluxo por meio da $\mathrm{FN}$ não foi totalmente efetivo para cessar o processo inflamatório na mucosa esofágica. Embora sem significância estatística $(p=0,1976)$, houve alteração do número de casos sem inflamação histológica após a FN. Antes desta, seis pacientes não tinham inflamação e 19 apresentavam inflamação histológica (11 ativa e oito crônica) e, após o procedimento cirúrgico, 11 pacientes não tinham inflamação histológica e14 tinham evidências de inflamação (cinco ativa e nove crônica). Cerca $20 \%$ dos nossos pacientes, inclusive, apresentaram recorrência dos sintomas clínicos da DRGE.

A incidência de displasia e adenocarcinoma em EB tem associação comprovada com o tempo de evolução dos sintomas de DRGE ${ }^{(28)}$. Em nosso estudo, 60\% dos pacientes com EB relatavam tempo de sintomas compatíveis com DRGE até o momento do diagnóstico (endoscópico- -histológico) em até cinco anos (média de 88 meses). $O$ diagnóstico de EB foi feito com períodos menores de relato de sintomas pelos pacientes (inferiores a cinco anos). A baixa prevalência de displasia e de adenocarcinoma por nós encontrada (24\%) pode estar associada a esse intervalo relativamente curto de sintomatologia.

Em um recente estudo, as áreas de mucosa metaplásica de Barrett com positividade para MUC1 mostraram maior associação ao refluxo de longa data em comparação com os casos de MUC1 negativo ${ }^{(13)}$. Ou seja, refluxos mais antigos tendem a positivar mais o marcador MUC1 em comparação com refluxos recentes. Nosso estudo mostrou positividade para MUC1 apenas no epitélio escamoso esofágico, com padrão de membrana e sem alterações da sua expressão antes e depois da correção do refluxo. Esses achados podem também estar associados ao intervalo curto de sintomas da DRGE. Não observamos nenhuma expressão de MUC1 no epitélio metaplásico, o que também pode estar relacionado com o baixo risco de carcinogênese.

O epitélio metaplásico pode refletir uma resposta adaptativa a um novo ambiente ácido por meio da expressão das mucinas MUC5AC e MUC6, que oferecem proteção ao suco gástrico, e da MUC2, relacionada com a proteção do refluxo de bile. Burjonrappa et al. ${ }^{(6)}$ destacam a diminuição da expressão dessas mucinas como um processo evolutivo para lesões pré-neoplásicas. Assim, a down-regulation poderia indicar diminuição dos mecanismos protetores da mucosa esofágica. Não observamos diferenças estatisticamente significativas quanto à imunoexpressão de MUC2, MUC5AC e MUC6 entre as biópsias antes e depois da FN, indicando, provavelmente, manutenção desses mecanismos protetores da mucosa e baixo risco de carcinogênese. Embora tenhamos observado redução da expressão desses marcadores nas áreas de displasia e adenocarcinoma, trata-se de apenas seis casos (24\%) e essas diferenças não foram, portanto, estatisticamente significantes.

Alguns estudos demonstram que em pacientes submetidos a correção cirúrgica de refluxo a metaplasia não foi mais detectada em biópsias pós-cirúrgicas de seguimento, não se observando expressão de CDX2 nesses $\operatorname{casos}^{(14,30)}$. Não verificamos alterações na imunoexpressão do CDX2 no epitélio metaplásico antes e depois da FN, o que provavelmente relaciona-se com a não eficácia da cirurgia em cessar o processo inflamatório causado pelo refluxo na mucosa esofágica. Por outro lado, também não observamos maior risco de carcinogênese associada à perda de expressão desse marcador nos casos estudados. 
Muitas das dificuldades de interpretação e, por vezes, resultados inconclusivos, provavelmente estão relacionados com uma casuística pequena e também com um intervalo relativamente curto de evolução da DRGE. Ressalta-se a necessidade de estudos com casuísticas maiores e com períodos mais longos de seguimento e de evolução dos sintomas de refluxo.

\section{Conclusão}

Os resultados permitem concluir que não houve diferenças significativas da presença ou do grau de inflamação esofágica e da imunoexpressão de MUC1, MUC2, MUC5AC, MUC6 e CDX2 no EB antes e após a FN. O tratamento cirúrgico não influenciou a mudança da expressão dessas glicoproteínas no EB.

A negatividade da MUC1 no EB antes e após a FN, assim como a manutenção da expressão de MUC2, MUC5AC, MUC6 e CDX2 podem indicar estabilidade da mucosa esofágica e também baixo risco de carcinogênese na população estudada, já que apenas $24 \%$ dos pacientes apresentavam displasia ou adenocarcinoma.

\section{Apoio}

Esta pesquisa contou com o apoio e o financiamento do Programa Institucional de Bolsas de Iniciação Científica PIBIC/ CNPq - vigência 01 de agosto de 2008 a 31 de julho de 2009.

\section{Referências}

1. ALMEIDA, R. et al. Expression of intestine-specific transcription factors, CDX1 and CDX2, in intestinal metaplasia and gastric carcinomas. J Pathol, v. 199, n.1, p. 36-40, 2003.

2. AMANO, Y. et al. Prevalence and risk factors for Barrett's esophagus with intestinal predominant mucin phenotype. Scand J Gastroenterol, v. 41, p. 873-9, 2006.

3. ANDREOLLO, N. A. et al. Incidência e epidemiologia do epitélio de Barrett no Gastrocentro/Unicamp. Arq Gastroenterol, v. 34, p. 22-6, 1997.

4. ARUL, G. S. et al. Mucin gene expression in Barrett's oesophagus: an in situ hybridization and immunohistochemical study. Gut, v. 47, p. 753-61, 2000.

5. BANKI, F. et al. Barrett's esophagus in females: a comparative analysis of risk factors in females and males. Am J Gastroenterol, v. 100, n. 3, p. 560-7, 2005.

6. BURJONRAPPA, S. C. et al. Mucin expression profile in Barret's, dysplasia, adenocarcionoma sequence in the esophagus. Indian J of Cancer, v. 44, n. 1, p. 1-5, 2007.

7. CAMERON, A. J. et al. Barrett's esophagus: age, prevalence, and extent of columnar epitelium. Gastroenterology, v. 103, n. 4, p. 1241-5, 1992.

8. CHALASANI, N. et al. Significance of intestinal metaplasia in different areas of esophagus including esophagogastric junction. Dig Dis Sci, v. 42, n. 3, p. 603-7, 1997.

9. CHINYAMA, C. N. et al. Expression of MUC1 and MUC2 mucin gene products in Barrett's metaplasia, dysplasia and adenocarcinoma an immunopathological study with clinical correlation. Histopathology, v. 35, p. 51724, 1999.

10. CORSI, P. R. et al. Factors related to the presence of reflux in patients with typical symptoms of gastroesophageal reflux disease (GERD). Rev Assoc Med Bras, v. 53, n. 2, p. 152-7, 2007.
11. FLÉJOU, J. F. Barrett's oesophagus: from metaplasia to dysplasia and cancer. Gut, v. 54, Suppl.1, p. 6-12, 2005.

12. FLUCKE, U. et al. Immunoreactivity of cytokeratins (CK7, CK20) and mucin peptide core antigens (MUC1, MUC2, MUC5AC) in adenocarcinomas, normal and metaplastic tissues of the distal oesophagus, oesophago-gastric junction and proximal stomach. Histopathology, v. 43, n. 2, p. 127-34, 2003.

13. GLICKMAN, J. N. et al. Mucin core polypeptide expression in the progression of neoplasia in Barrett's esophagus. Hum Pathol, v. 37, p. 1304-15, 2006.

14. GLICKMAN, J. N.; SHAHSAFAEI, A.; ODZE, R. D. Mucin core peptide expression can help differentiate Barrets esophagus from intestinal metaplasia of the stomach. Am J Surg Pathol, v. 27, n. 10, p. 1357-65, 2003.

15. JONES, P. T. General practice in London. Br J Gen Pract, v. 40, n. 340, p. 480, 1990.

16. KHOR, S. T. et al. Divergent expression of MUC5AC, MUC6, MUC2, CD10, and CDX2 in dysplasia and intramucosal adenocarcinomas with intestinal and foveolar morphology: is this evidence of distinct gastric and intestinal pathways to carcinogenesis in Barrett esophagus? Am J Surg Pathol, v. 36, p. 331-42, 2012.

17. MGROISMAN, G.; AMAR, M.; MEIR, A. Expression of the intestinal marker CDX2 in the columnar lined esophagus with and without intestinal (Barrett's) metaplasia. Modern Pathology, v. 17, n. 10, p. 1282-8, 2004.

18. MOONS, L. M. et al. The homeodomain protein CDX2 is an early marker of Barrett's oesophagus. J Clin Pathol, v. 57, n. 10, p. 1063-8, 2004.

19. MORAES-FILHO J. et al. Brazilian consensus on gastroesophageal reflux disease: proposals for assessment, classification, and management. Am J Gastroenterol, v. 97, p. 241-8, 2002. 
20. MOYES, L. H.; GOING J. J. Still waiting for predictive biomarkers in Barrett's oesophagus. J Clin Pathol, v. 64, p. 742-50, 2011.

21. NASI, A. et al. Doença do refluxo gastroesofágico: comparação entre as formas com e sem esofagite, em relação aos dados demográficos e às manifestações sintomáticas. Arq Gastroenterology, v. 38, n. 2, p. 109-15, 2001.

22. NASI, A. et al. Doença do refluxo gastroesofágico: revisão ampliada. Arq Gastroenterol, v. 43, p. 334-41, 2006.

23. NIVE, Y.; FASS, R. The role of mucin in GERD and its complications. Nat Rev Gastroenterol Hepatol, v. 9, p. 55-9, 2012.

24. Park, Y. et al. CDX2 expression in the intestinal-type gastric epithelial neoplasia: frequency and significance. Mod Pathol, v. 23, p. 54-61, 2010.
25. PASCU, O.; LENCU, M. Barrett's esophagus. Rom J Gastroenterol, v. 13, n. 3, p. 219-22, 2004.

26. PIÉROLA, B. F. Controversias en el esófago de Barrett. An Med Interna, v. 18, p. 507-9, 2001.

27. RONKAINEN, J. et al. Prevalence of Barretts esophagus in the general population: an endoscopic study. Gastroenterology, v. 129, p. 1825-31, 2005.

28. SPECHLER, S. J. et al. Prevalence of metaplasia at the gastro-oesophageal junction. Lancet, v. 344, n. 8936, p. 1533-6, 1994.

29. VAN SANDICK, J. W. et al. Intestinal metaplasia of the esophagus or esophagogastric junction: evidence of distinct clinical, pathologic, and histochemical staining features. Am J Clin Pathol, v. 117, n. 1, p. 117-25, 2002.

30. ZANINOTTO, G. et al. Barrett's epithelium after antireflux surgery. J Gastrointest Surg, v. 9, n. 9, p. 1253-60, 2005. 